\title{
The gum health game-changer
}

AIRFLOW Prophylaxis Master is the latest EMS innovation for guided biofilm therapy (GBT), a unique solution for periodontal disease prevention and maintenance.

Created for intensive professional use, the device is characterised by unique ergonomics, high precision, easy maintenance, and compliance with the highest hygiene standards.

The AIRFLOW Prophylaxis Master has been designed with the highest standards of performance, safety and comfort in mind, being specifically built to deliver the three core treatments of GBT.

GBT is the systematic, predictable solution for dental biofilm management in professional prophylaxis using state-of-theart AIRFLOW, PERIOFLOW and PIEZON technologies. It is proven by scientific evidence.

1. AIRFLOW handpiece removes biofilm, stains and young calculus on natural teeth, restorations and implants. Cleans and polishes in one single procedure

2. PERIOFLOW Handpiece and PLUS powder are indicated for:

- Subgingival biofilm removal (debridement) in $>4$ to $9 \mathrm{~mm}$ periodontal and peri-implant pockets

- Initial and follow-up (SPT) periodontal therapy

- Prevention of mucositis/ peri-implantitis
- Initial and follow-up treatment of mucositis/peri-implantitis

3. PIEZON Handpiece removes remaining supra and subgingival calculus with the PIEZON NO PAIN PS instrument.

The GBT protocol is based on individual patient diagnosis and risk assessment in order to achieve optimal results. The treatment is given in the least invasive way, with the highest level of comfort, safety and efficiency.

If you would like further details about what EMS Dental has to offer dental professionals in the UK, visit www.emsdental.com.

\section{Focusing on the here and now}

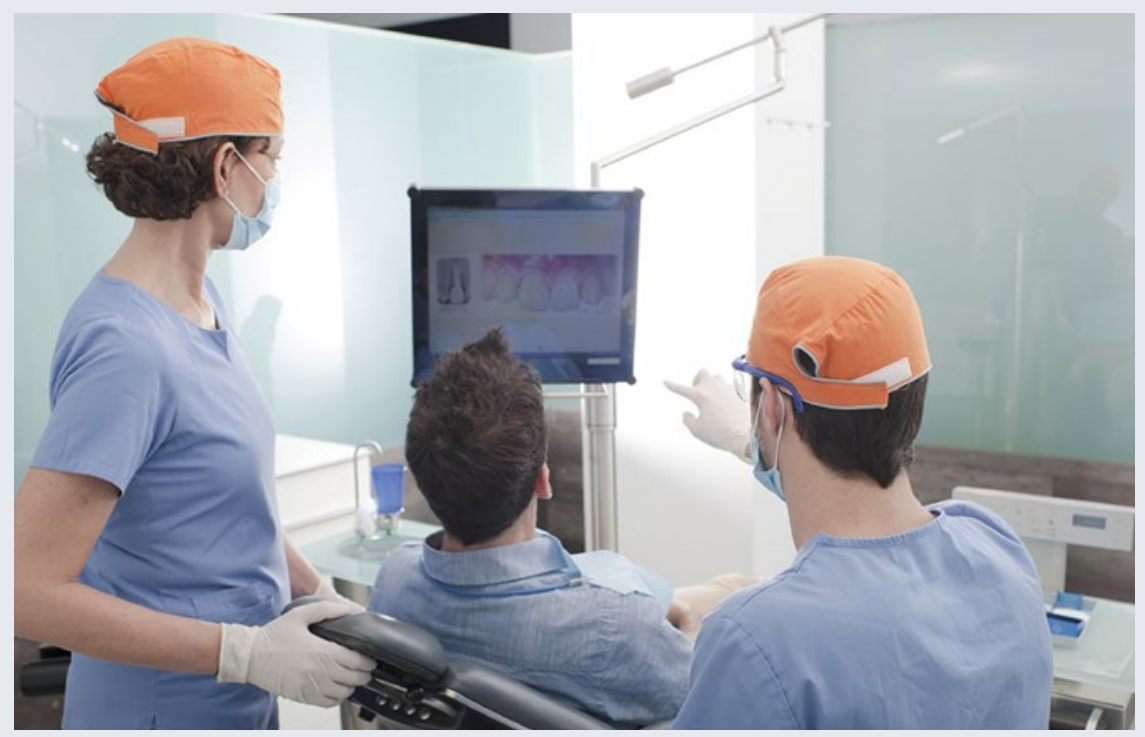

The British Dental Conference and Dentistry Show has been postponed so that you can focus on your practice and your patients. The next event will be held on Friday 21 and Saturday 22 May 2021 in Birmingham, allowing time for the dust to settle before helping you to move forward.

When the time comes, you can expect everything the conference is well-known for, including a diverse educational programme, eCPD for the entire team, world-class speakers, an extensive trade show and copious networking opportunities.

Right now, the organisers are offering all members of the team free access to the popular and growing 'Back to Practice' webinar library to help you re-open the doors successfully. A monthly e-bulletin also offers advice and guidance from key individuals and organisations within the profession sign up at www.thedentistryshow.co.uk/ newsletter-signup.

The next British Dental Conference and Dentistry Show will be held on Friday 21 and Saturday 22 May 2021, Birmingham NEC, co-located with DTS.

For more information, visit www. thedentistryshow.co.uk, call 02073485270 or email dentistry@closerstillmedia.com.

\section{Revolutionise your bonding}

While you're updating and enhancing your clinical processes to adapt to the 'new normal', revolutionise your bonding with the APC Flash-Free Adhesive Coated Appliance System from 3M Oral Care.

You and your patients will benefit from a faster bond-up procedure that ensures a stronger bond between bracket and tooth for more reliable and predictable treatment progress. In addition, it eliminates the need for flash clean-up, as required with traditional bracket adhesives for greater efficiency and a simpler workflow.

Find out more from the team at $3 \mathrm{M}$ Oral Care today.

For more information, call 08705360036 or visit www. 3 m.co.uk/apcff.

$3 \mathrm{M}$ and Incognito are trademarks of the 3M Company.

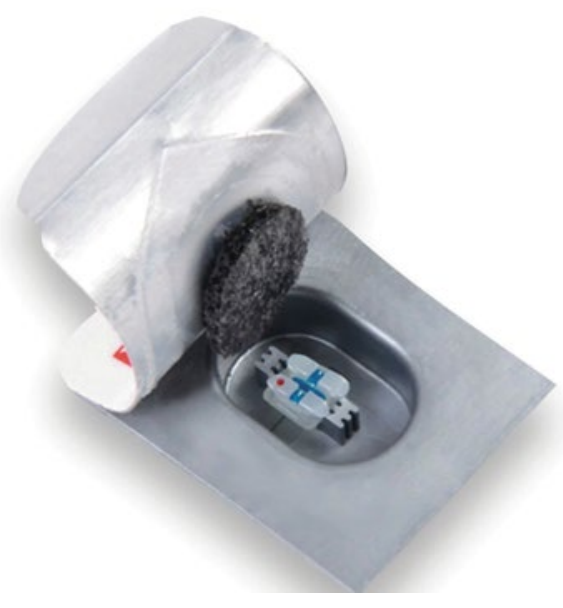

